\title{
ANTIPALINDROMIC NUMBERS
}

\author{
L'ubomíra Dvořákováa,*, Stanislav Kruml ${ }^{a}$, David RyzÁK ${ }^{b}$ \\ ${ }^{a}$ Czech Technical University in Prague, Faculty of Nuclear Sciences and Physical Engineering, Department of \\ Mathematics, Trojanova 13, 12000 Praha 2, Czech Republic \\ ${ }^{b}$ Charles University, Faculty of Mathematics and Physics, Ke Karlovu 2027, 12116 Praha 2, Czech Republic \\ * corresponding author: lubomira.dvorakova@fjfi.cvut.cz
}

\begin{abstract}
Everybody has certainly heard about palindromes: words that stay the same when read backwards. For instance, kayak, radar, or rotor. Mathematicians are interested in palindromic numbers: positive integers whose expansion in a certain integer base is a palindrome. The following problems are studied: palindromic primes, palindromic squares and higher powers, multi-base palindromic numbers, etc. In this paper, we define and study antipalindromic numbers: positive integers whose expansion in a certain integer base is an antipalindrome. We present new results concerning divisibility and antipalindromic primes, antipalindromic squares and higher powers, and multi-base antipalindromic numbers. We provide a user-friendly application for all studied questions.
\end{abstract}

KEYWORDS: Expansion in a base, palindromes, antipalindromes, palindromic numbers, antipalindromic numbers.

\section{INTRODUCTION}

Everybody has certainly heard about palindromes: words that stay the same when read backwards. For instance, kayak, radar, or rotor. It is not surprising that in natural languages, it is impossible to find extremely long palindromes. The longest palindrome in English is "tattarrattat". However its victory is doubtful since tattarrattat is a neologism created by James Joyce in his novel Ulysses [1] it expresses loud knocking at the door:

"I was just beginning to yawn with nerves thinking he was trying to make a fool of me when I knew his tattarrattat at the door."

Palindromic phrases are even more interesting. They provide palindromes if punctuation, capitalization, and spaces are ignored. Some popular palindromic phrases (the last one devoted to mathematicians) in English are:

"Do geese see God?"

"A man, a plan, a canal: Panama."

"Madam, in Eden, I'm Adam."

"never odd or even"

Mathematicians are interested in palindromic numbers: positive integers whose expansion in a certain integer base is a palindrome. Let us make a list of studied problems:

(1.) Palindromic squares, cubes, and higher powers in base 10: The first nine terms of the sequence $1^{2}, 11^{2}, 111^{2}, 1111^{2}, \ldots$ are palindromic numbers $1,121,12321,1234321, \ldots$ (sequence A002477 in the OEIS [2]). Palindromic numbers having palindromic squares were studied in [3]. The only known nonpalindromic number whose cube is a palindromic number is 2201, Simmons [4] conjectured that the fourth root of all palindromic fourth powers are palindromic numbers of the form $10^{n}+1$. Simmons [5] also conjectured there are no palindromic numbers of the form $n^{k}$ for $k>4$ and $n>1$.

(2.) Palindromic primes: The first few decimal palindromic primes are (sequence A002385 in the OEIS [2]):

$$
2,3,5,7,11,101,131,151,181,191,313,353 \text {, }
$$

$$
373,383,727,757,787,797,919,929, \ldots
$$

Except for 11, all palindromic primes have an odd number of digits because the divisibility test for 11 tells us that every palindromic number with an even number of digits is divisible by 11 . On the one hand, it is not known if there are infinitely many palindromic primes in base 10; the largest known decimal palindromic prime has 474,501 digits (found in 2014):

$$
10^{474500}+999 \cdot 10^{237249}+1 \text {. }
$$

On the other hand, it is known that, for any base, almost all palindromic numbers are composite [6]. It means that the ratio of palindromic composites and all palindromic numbers less than $n$ tends to 1 .

Binary palindromic primes include the Mersenne primes and the Fermat primes ${ }^{1}$. All binary palindromic primes, except the number 3 (having the expansion $\overline{11}$ in base 2 ), have an odd number of digits; palindromic numbers with an even number of digits are divisible by 3 . Let us write down

\footnotetext{
${ }^{1} \mathrm{~A}$ Mersenne prime is a prime of the form $2^{p}-1$, where $p$ is a prime. A Fermat prime is a prime of the form $2^{2^{n}}+1$.
} 
the sequence of binary expansions of the first binary palindromic primes (sequence A117697 in the OEIS [2]):

$$
\begin{aligned}
& \overline{11}, \overline{101}, \overline{111}, \overline{10001}, \overline{11111}, \overline{1001001}, \overline{1101011}, \\
& \overline{1111111}, \overline{100000001}, \overline{100111001}, \overline{110111011}, \ldots
\end{aligned}
$$

(3.) Multi-base palindromic numbers: Any positive integer $n$ is palindromic in all bases $b$ with $b \geq n+1$ because $n$ is then a single-digit number, and also in base $n-1$ because the expansion of $n$ in base $n-1$ equals $\overline{11}$. But, it is more interesting to consider bases smaller than the number itself. For instance, the number 105 is palindromic in bases $4,8,14,20,34,104$; the expansions of 105 in those bases are:

$$
\begin{aligned}
& (105)_{4}=\overline{1221}, \quad(105)_{8}=\overline{151}, \quad(105)_{14}=\overline{77}, \\
& (105)_{20}=\overline{55}, \quad(105)_{34}=\overline{33}, \quad(105)_{104}=\overline{11} .
\end{aligned}
$$

A palindromic number in base $b$ whose expansion is made up of palindromic sequences of length $\ell$ arranged in a palindromic order is palindromic in base $b^{\ell}$. For example, the number 24253 has the expansion in base 2 equal to $(24253)_{2}=$ 101111010111101 , i.e., it is made up of palindromes of length of 3 , and its expansion in base $2^{3}=8$ is equal to $(24253)_{8}=\overline{57275}$.

(4.) Sum of palindromes: Every positive integer can be written as the sum of at most three palindromic numbers in every number system except the binary system. It was proven first for bases greater than or equal to 5 in 7 and for bases 3,4 in 8. Moreover, the optimal bound for base 2 is four, see [8].

In this paper, we deal with antipalindromic numbers in various integer bases. We examine and compare properties of palindromic numbers and antipalindromic numbers, bringing a number of new results. These are structured as follows. In Section 2 , we introduce the definition of an antipalindromic number in an integer base and its basic properties, following from the definition. Section 3 brings unexpected results concerning divisibility and antipalindromic primes. In Section 4 antipalindromic squares and higher powers are examined. Section 5 contains information about numbers that are antipalindromic in two or more bases at the same time. In Section 6, we summarize our results and provide a list of conjectures and open problems.

\section{DeFinition AND BASIC PROPERTIES}

Let us start with a formal definition of palindromic and antipalindromic numbers and their basic properties.

Definition 1. Let $b \in \mathbb{N}, b \geq 2$. Consider a natural number $m$ whose expansion in base $b$ is of the following form

$$
m=a_{n} b^{n}+\cdots+a_{1} b+a_{0},
$$

where $a_{0}, a_{1}, \ldots, a_{n} \in\{0,1, \ldots, b-1\}, a_{n} \neq 0$. We usually write $(m)_{b}=\overline{a_{n} \ldots a_{1} a_{0}}$. Then $m$ is called
(1.) a palindromic number in base $b$ if its digits satisfy the condition:

$$
a_{j}=a_{n-j} \quad \text { for all } j \in\{0,1, \ldots, n\},
$$

(2.) an antipalindromic number in base $b$ if its digits satisfy the condition:

$$
a_{j}=b-1-a_{n-j} \quad \text { for all } j \in\{0,1, \ldots, n\} .
$$

The length of the expansion of the number $m$ is usually denoted $|m|$.

Example 1. Consider distinct bases $b$ and have a look at antipalindromic numbers in these bases:

- 395406 is an antipalindromic number in base $b=10$.

- $(1581)_{3}=\overline{2011120}$, i.e., 1581 is an antipalindromic number in base $b=3$.

- $(52)_{2}=\overline{110100}$, i.e., 52 is an antipalindromic number in base $b=2$.

Observation 1. If an antipalindromic number in base $b$ has an odd number of digits, then $b$ is an odd number and the middle digit is equal to $\frac{b-1}{2}$.

Proof. It follows from the definition that twice the middle digit is equal to $b-1$. Thus, $b$ is an odd number and the middle digit is equal to $\frac{b-1}{2}$.

Observation 2. A number is simultaneously palindromic and antipalindromic if and only if $b$ is an odd number and all the digits are equal to $\frac{b-1}{2}$.

Proof. Consider an antipalindromic number with digits $a_{0}, a_{1}, \ldots, a_{n}$. For this number to be palindromic, $a_{j}=a_{n-j}$ must be true for each $j \in\{0,1, \ldots, n\}$. From the definition of an antipalindromic number, it follows that $a_{j}+a_{n-j}=b-1$. For each $j \in$ $\{0,1, \ldots, n\}$, we obtain $a_{j}=\frac{b-1}{2}$, i.e., all digits are equal to $\frac{b-1}{2}$ and the base $b$ must, therefore, be odd. The opposite implication is obvious.

\section{DivisibILITY AND ANTIPALINDROMIC PRIMES}

Let us first study the divisibility of antipalindromic numbers, which will be used in the sequel to show interesting results on antipalindromic primes.

Lemma 1. Let $m$ be a natural number and its expansion in base $b$ be equal to $a_{n} b^{n}+a_{n-1} b^{n-1}+$ $\ldots+a_{1} b+a_{0}$. Then $m$ is divisible by $b-1$ if and only if the sum of its digits is divisible by $b-1$, i.e., $a_{n}+a_{n-1}+\ldots+a_{1}+a_{0} \equiv 0(\bmod b-1)$.

Proof. The statement follows from the fact that $b^{k} \equiv 1(\bmod b-1)$ for any $k \in \mathbb{N}$.

Theorem 2. Any antipalindromic number with an even number of digits in base $b$ is divisible by $b-1$. 
Proof. Consider an antipalindromic number

$$
m=a_{n} b^{n}+a_{n-1} b^{n-1}+\ldots+a_{1} b+a_{0}
$$

for an odd $n$. From the definition, it is true that $a_{j}+a_{n-j}=b-1$ for each $j \in\{0,1, \ldots, n\}$. The number of digits is even, hence

$a_{n}+a_{n-1}+\ldots+a_{1}+a_{0}=(b-1) \frac{n+1}{2} \equiv 0(\bmod b-1)$.

Using Lemma 1. the antipalindromic number $m$ is divisible by the number $b-1$.

Theorem 3. An antipalindromic number with an odd number of digits in base $b$ is divisible by $\frac{b-1}{2}$.

Proof. Consider the antipalindromic number

$$
m=a_{2 n} b^{2 n}+a_{2 n-1} b^{2 n-1}+\ldots+a_{1} b+a_{0} .
$$

The digit sum of the number $m-a_{n} b^{n}$ is divisible by $b-1$. From Lemma 1, we also know that the number $m-a_{n} b^{n}$ itself is divisible by $b-1$ and, therefore, by $\frac{b-1}{2}$. From Observation 1 $a_{n}=\frac{b-1}{2}$. The number $m$ is a sum of two numbers divisible by $\frac{b-1}{2}$.

Let us now turn our attention to antipalindromic primes. While palindromic primes occur in various bases, antipalindromic primes occur (except some trivial cases) only in base 3 .

Theorem 4. Let base $b>3$. Then there exists at most one antipalindromic prime number $p$ in base $b$ : $p=\frac{b-1}{2}$.

Proof. Theorems 2 and 3 show that every antipalindromic number is divisible either by $\frac{b-1}{2}$ or $b-1$. Although $b-1$ may be a prime number, it is never antipalindromic.

Theorem 5. Let base $b=2$. Then there exists only one antipalindromic number $p=2,(p)_{2}=\overline{10}$.

Proof. Every antipalindromic number in base $b=2$ is even. 2 is the only even prime number.

Theorem 6. Let base $b=3$. Every antipalindromic prime in this base has an odd number of digits $n \geq 3$.

Proof. From Theorem 2, antipalindromic numbers with an even number of digits in base $b=3$ are even. The only antipalindromic number in this base with one digit is 1 .

Lemma 7. Antipalindromic numbers in base $b=3$ beginning with the digit 2 are divisible by 3 .

Proof. Consider an antipalindromic number $m=$ $a_{n} 3^{n}+a_{n-1} 3^{n-1}+\ldots+a_{1} 3+a_{0}$, where $a_{n}=2$. The sum of $a_{n}$ and $a_{0}$ need to be equal to 2 , therefore, $a_{0}=0$. All the summands are divisible by 3 .

Theorem 8. All antipalindromic primes in base $b=3$ can be expressed as $6 k+1$, where $k \in \mathbb{N}$.
Proof. Consider an antipalindromic prime $m=$ $a_{2 n} 3^{2 n}+a_{2 n-1} 3^{2 n-1}+\ldots+a_{1} 3+a_{0}$. (The number of digits must be odd.) From Lemma 7, $a_{0}$ is equal to 1 and $a_{2 n}=1$. Let us pair the digits of the antipalindromic number $m$ (except for $a_{2 n}, a_{n}$, and $\left.a_{0}\right): a_{2 n-j} 3^{2 n-j}+a_{j} 3^{j}, j \in\{1, \ldots, n-1\}$. Let us prove that for each $j \in\{1, \ldots, n-1\}$, the following expression is divisible by 6

$$
3^{j}\left(a_{2 n-j} 3^{2 n-2 j}+a_{j}\right)
$$

We can only consider three possibilities: $a_{2 n-j}=$ $2, a_{j}=0$, or $a_{2 n-j}=a_{j}=1$, or $a_{2 n-j}=0, a_{j}=2$. In either case, the number in question is divisible by 6 because there is an even number inside the bracket. We then get

$$
\begin{aligned}
m & =a_{2 n} 3^{2 n}+a_{n} 3^{n}+a_{0}+6 \ell \\
& =3^{2 n}+3^{n}+1+6 \ell \\
& =3^{n}\left(3^{n}+1\right)+1+6 \ell
\end{aligned}
$$

for some $\ell \in \mathbb{N}$. The first summand is also divisible by 6 , therefore $m$ can indeed be expressed as $6 k+1$ for some $k \in \mathbb{N}$.

The application 9 can be used for searching antipalindromic primes in base 3. During an extended search, the first 637807 antipalindromic primes have been found. Let us now list at least the first 10 of them, along with their expansions in base 3 :

$\begin{array}{rr}13 & \overline{111} \\ 97 & \overline{10121} \\ 853 & \overline{1011121} \\ 1021 & \overline{1101211} \\ 1093 & \overline{1111111} \\ 7873 & \overline{101210121} \\ 8161 & \overline{102012021} \\ 8377 & \overline{102111021} \\ 9337 & \overline{110210211} \\ 12241 & \overline{121210101}\end{array}$

\section{SquARES AND OTHER POWERS AS ANTIPALINDROMES}

For palindromic numbers, squares and higher powers were considered in 4, 5, by Simmons more than thirty years ago. He proved that there were infinitely many palindromic squares, cubes, and biquadrates. However, his conjecture was that for $k>4, k \in \mathbb{N}$, no integer $m$ exists such that $m^{k}$ is a palindromic number (in the decimal base). This conjecture is still open. That is definitely not the case for antipalindromic numbers as $3^{7}=2187$ is antipalindromic in base 10 . For a more recent study of palindromic powers, see 10.

Let us answer the following question: 


\begin{tabular}{ccccccc}
\hline base & $\mathrm{n}=20$ & 21 & 22 & 23 & 24 & 25 \\
\hline$n^{2}$ & 3 & 13 & 3 & 14 & 9 & 11 \\
$n^{2}+1$ & 47 & 44 & 48 & 53 & 55 & 57 \\
$n^{2}+2$ & 2 & 2 & 2 & 2 & 2 & 1 \\
\hline
\end{tabular}

TABLE 1. Number of antipalindromic squares smaller than $10^{12}$ in particular bases

\begin{tabular}{cccccccccc}
\hline base & $\mathrm{n}=4$ & 5 & 6 & 7 & 8 & 9 & 10 & 11 & 12 \\
\hline$n^{4}$ & 0 & 1 & 0 & 1 & 0 & 1 & 0 & 0 & 0 \\
$n^{4}+1$ & 6 & 6 & 8 & 10 & 13 & 13 & 13 & 13 & 13 \\
$n^{4}+2$ & 0 & 0 & 0 & 0 & 0 & 0 & 0 & 0 & 0 \\
\hline
\end{tabular}

TABle 2. Number of antipalindromic biquadrates smaller than $10^{15}$ in particular bases

Question 1. Are there any antipalindromic integer squares?

Our initial observation suggested that bases $b=$ $n^{2}+1, n \in \mathbb{N}$, have the most antipalindromic squares and the computer application provided an additional insight needed to prove this observation not only for squares but for other powers as well. Table 1 expresses the number of antipalindromic squares smaller than $10^{12}$ in bases $n^{2}, n^{2}+1$, and $n^{2}+2$ to underline the differences between the bases of the form $n^{2}+1$ and the others.

As the exponent of $n^{k}$ is raised, the differences between the number of antipalindromic $k$-powers in the base $n^{k}+1$ and the others become even more significant, but the numbers rise faster, thus we meet the computational limits of our program already for small values of $n$, see Table 2 .

Example 2. Consider the base $b=10=3^{2}+1$. Any antipalindromic number in this base must be divisible by 9. Every double-digit number divisible by 9 (except 99) is antipalindromic:

$$
18,27,36,45,54,63,72,81,90 .
$$

The number 9 is a square, so if a square is divided by 9 , it is still a square.

$$
36=4 \cdot 9=2^{2} \cdot 3^{2}=6^{2}, \quad 81=9 \cdot 9=9^{2} .
$$

Thus 36 and 81 are antipalindromic squares.

Proposition 1. For $b=n^{2}+1, n \in \mathbb{N}$, and $m \in$ $\{2,3, \ldots, n\}$, the number $(m \cdot n)^{2}$ is antipalindromic.

Proof. Since $b=n^{2}+1$, we can modify the expression as follows: $(m \cdot n)^{2}=m^{2} \cdot(b-1)$. This number has the expansion in base $b$ equal to $\overline{\left(m^{2}-1\right)\left(b-m^{2}\right)}$, hence it is antipalindromic.

Further on, we will answer the following question.

Question 2. Are there any higher integer powers that are also antipalindromic numbers?
Example 3. Consider the base $b=28=3^{3}+1$. Any antipalindromic number in this base with an even number of digits must be divisible by 27. Every double-digit number divisible by 27 (except the one with expansion $\overline{(27)(27)}$ ) is antipalindromic.

The number 27 is a third power of 3 , so if a third power of any number is divided by 27 , it still is a third power of an integer.

$$
\begin{aligned}
& \overline{(7)(20)}=(216)_{28} \text { and } 216=8 \cdot 27=2^{3} \cdot 3^{3}=6^{3}, \\
& \overline{(26)(1)}=(729)_{28} \text { and } 729=27 \cdot 27=3^{3} \cdot 3^{3}=9^{3} .
\end{aligned}
$$

Thus 216 and 729 are antipalindromic cubes.

Theorem 9. For $b=n^{k}+1$, where $n, k \in \mathbb{N}, k \geq 2$, and $m \in\{2,3, \ldots, n\}$, the number $(m \cdot n)^{k}$ is antipalindromic.

Proof. Since $b=n^{k}+1$, we can modify the expression as follows: $(m \cdot n)^{k}=m^{k} \cdot(b-1)$. This number has the expansion in base $b$ equal to $\overline{\left(m^{k}-1\right)\left(b-m^{k}\right)}$, thus it is antipalindromic.

For odd powers and high enough bases, other patterns exist.

Theorem 10. For integers $m>1$ and odd $k>1$, there exists a number $c$ such that in every base $b \geq c$, the following number is antipalindromic:

$$
[m \cdot(b-1)]^{k} \text {. }
$$

It suffices to put $c=\left(\begin{array}{c}k \\ \frac{k-1}{2}\end{array}\right) \cdot m^{k}$.

Proof. The binomial theorem reads

$$
[m \cdot(b-1)]^{k}=m^{k} \cdot \sum_{i=0}^{k}(-1)^{i} \cdot\left(\begin{array}{c}
k \\
i
\end{array}\right) \cdot b^{k-i} .
$$

Since $\left(\begin{array}{c}k \\ \frac{k-1}{2}\end{array}\right)$ is the maximum number among $\left(\begin{array}{c}k \\ i\end{array}\right)$ for $i \in\{0,1, \ldots, k\}$, the expansion in base $b$ equals: $\left([m \cdot(b-1)]^{k}\right)_{b}=$ $=\overline{\left(m^{k} \cdot\left(\begin{array}{c}k \\ 0\end{array}\right)-1\right)\left(b-m^{k} \cdot\left(\begin{array}{l}k \\ 1\end{array}\right)\right)\left(m^{k} \cdot\left(\begin{array}{l}k \\ 2\end{array}\right)-1\right) \ldots}$ $\overline{\ldots\left(b-m^{k} \cdot\left(\begin{array}{c}k \\ k-2\end{array}\right)\right)\left(m^{k} \cdot\left(\begin{array}{c}k \\ k-1\end{array}\right)-1\right)\left(b-m^{k} \cdot\left(\begin{array}{l}k \\ k\end{array}\right)\right)}$.

\section{Multi-BASE ANTIPALINDROMiC NUMBERS}

Let us study the question whether there are numbers that are antipalindromic simultaneously in more bases. Bašić in [11, 12] showed that for any $n \in \mathbb{N}$ and any $d \geq 2$, there exists an integer $m$ and a list of $n$ bases such that $m$ is a $d$-digit palindromic number in each of the bases. We do not know whether something similar holds for antipalindromic numbers. We will show a weaker statement that for any $n \in \mathbb{N}$, there exists an integer $m$ and a list of $n$ bases such that $m$ is antipalindromic in each of the bases. 


\begin{tabular}{cc}
\hline base & expansion \\
\hline 2 & $\overline{110011001100}$ \\
4 & $\overline{303030}$ \\
10 & $\overline{3276}$ \\
64 & $\overline{(53)(10)}$ \\
79 & $\overline{(41)(37)}$ \\
85 & $\overline{(38)(46)}$ \\
92 & $\overline{(27)(90)}$ \\
118 & $\overline{(25)(101)}$ \\
127 & $\overline{(20)(136)}$ \\
157 & $\underline{(17)(165)}$ \\
183 & $\underline{(13)(221)}$ \\
235 & $\underline{(12)(240)}$ \\
253 & $\overline{(11)(262)}$ \\
274 & $\underline{(6)(356)}$ \\
365 & $\underline{(5)(541)}$ \\
469 & $\underline{(3)(816)}$ \\
547 & $\underline{(2)(1090)}$ \\
820 & $(1637)$ \\
1093 & \\
1639 & $(3276)$ \\
6553 & \\
\hline
\end{tabular}

TABLE 3. Antipalindromic expansions of the number 3276 in 21 bases

In 2014, Bérczes and Ziegler 13 discussed multibase palindromic numbers and proposed a list of the first 53 numbers palindromic in bases 2 and 10 simultaneously. Our application [9] has only been able to find one number with an antipalindromic expansion in these bases. This number, 3276, is also antipalindromic in other 19 distinct bases, see Table 3 . The next greater number that is antipalindromic both in base 2 and 10 must be greater than $10^{10}$ and divisible by 18 .

It is not uncommon for a number to be antipalindromic in more bases. In this section, we show that if a number is antipalindromic in a unique base, then the number must be prime or equal to 1 , see Theorem 11

Definition 2. An antipalindromic number is called multi-base if it is antipalindromic in at least two different bases.

Observation 3. Every number $m \in \mathbb{N}$ is antipalindromic in base $2 m+1$.

Example 4. The number 3276 is a multi-base antipalindromic number, as illustrated in Table 3 .

Theorem 11. For any composite number $a \in \mathbb{N}$, we can find at least two bases $b, c$ such that this number has an antipalindromic expansion in both of them.

Proof. Assume that $a=m \cdot n, m, n \in \mathbb{N}, m \geq n \geq 2$, set $b=\frac{a}{n}+1, c=2 a+1$. The expansions of $a$ in bases $b, c$ are equal to:

$$
\begin{aligned}
& (a)_{b}=\overline{(n-1)\left(\frac{a}{n}-n+1\right)}, \\
& (a)_{c}=\bar{a} .
\end{aligned}
$$

Theorem 12. For every $n \in \mathbb{N}$, there exist infinitely many numbers that are antipalindromic in at least $n$ bases.

Proof. Consider a number $a$ such that $a=(2 n)$ !. Theorem 11 indicates that the number $a$ is antipalindromic in bases $\frac{a}{2}+1, \frac{a}{3}+1, \ldots, \frac{a}{n}+1$ and also $2 a+1$.

Theorem 13. Let $b \in \mathbb{N}, b \geq 2$. Then there exists $m \in \mathbb{N}$ such that $m$ is antipalindromic in base $b$ and in at least one more base less than $\mathrm{m}$.

Proof.

$$
\begin{array}{lll}
\text { base } b & m & \text { two expansions } \\
2 & 12 & (12)_{2}=\overline{1100} \\
& & (12)_{4}=\overline{30} \\
3 & 72 & (72)_{3}=\overline{2200} \\
& & (72)_{9}=\overline{80} \\
\geq 4 & 4 \cdot(b-1) & (m)_{b}=\overline{(3)(b-4)} \\
& & (m)_{2 b-1}=\overline{(1)(2 b-3)}
\end{array}
$$

Theorem 14. Let $p, q \in \mathbb{N}$ such that $\operatorname{gcd}(p, q)=d$ and $p \geq \frac{q}{d}>1, q \geq \frac{p}{d}>1$. Then the number $m=\frac{p q}{d}$ is antipalindromic in bases $p+1$ and $q+1$.

Proof. We have

$$
\begin{aligned}
& (m)_{p+1}=\overline{\left(\frac{q}{d}-1\right)\left(p+1-\frac{q}{d}\right)}, \\
& (m)_{q+1}=\overline{\left(\frac{p}{d}-1\right)\left(q+1-\frac{p}{d}\right)} .
\end{aligned}
$$

Example 5. Let $p=4, q=6$, then $\operatorname{gcd}(4,6)=2$. The number $m=12$ is antipalindromic in bases 5 and $7:(12)_{5}=\overline{22},(12)_{7}=\overline{15}$.

In the introduction part, we have mentioned that a palindromic number in base $b$ whose expansion is made up of palindromic sequences of length $\ell$ arranged in a palindromic order is palindromic in base $b^{\ell}$. Let us present a similar statement for antipalindromic numbers. For its proof, we will need the following definition.

Definition 3. Let $b \in \mathbb{N}, b \geq 2$. Consider a string $u=u_{0} u_{1} \ldots u_{n}$, where $u_{i} \in\{0,1, \ldots, b-1\}$. The antipalindromic complement of $u$ in base $b$ is $A(u)=\left(b-1-u_{n}\right)\left(b-1-u_{n-1}\right) \ldots\left(b-1-u_{0}\right)$.

Theorem 15. Let $b \in \mathbb{N}, b \geq 2$. An antipalindromic number $m$ in base $b^{n}$, where $(m)_{b^{n}}=\overline{u_{k} \ldots u_{1} u_{0}}$ and $u_{k} \geq b^{n-1}$, is simultaneously antipalindromic in base $b$ if and only if the expansion of $u_{j}$ in base $b$ of length $n$ (i.e., completed with zeroes if necessary) is a palindrome for all $j \in\{0,1, \ldots, k\}$. 
Proof. The digits of $m$ in base $b^{n}$ satisfy $0 \leq u_{j} \leq$ $b^{n}-1$. Let us denote the expansion of $u_{j}$ in base $b$ by $\left(u_{j}\right)_{b}=\overline{v_{j, n-1} \cdots v_{j, 1} v_{j, 0}}$ (where the expansion of $u_{j}$ in base $b$ is completed with zeroes in order to have the length $n$ if necessary). The antipalindromic complement $A\left(u_{j}\right)$ of $u_{j}$ in base $b^{n}$ equals $b^{n}-1-$ $u_{j}$ and its expansion in base $b$ equals $\left(A\left(u_{j}\right)\right)_{b}=$ $\left(b-1-v_{j, n-1}\right) \ldots\left(b-1-v_{j, 1}\right)\left(b-1-v_{j, 0}\right)$. Since $m$ is antipalindromic in base $b^{n}$, we have $u_{k-j}=$ $A\left(u_{j}\right)=b^{n}-1-u_{j}$ for all $j \in\{0,1, \ldots, k\}$.

Let us now consider the expansion of $m$ in base $b$ : it is obtained by concatenation of the expansions of $u_{j}$ in base $b$ for $j \in\{0,1, \ldots, k\}$, i.e.,

$$
(m)_{b}=\overline{\left(u_{k}\right)_{b} \ldots\left(u_{1}\right)_{b}\left(u_{0}\right)_{b}} .
$$

Following the assertion that $u_{k} \geq b^{n-1}$, the expansion $\left(u_{k}\right)_{b}$ starts in a non-zero. Thus, the length of the expansion $(m)_{b}$ equals $n \cdot\left|(m)_{b^{n}}\right|$.

The number $m$ is antipalindromic in base $b$ if and only if $\left(u_{k-j}\right)_{b}=A\left(\left(u_{j}\right)_{b}\right)$ for all $j \in\{0,1, \ldots, k\}$, i.e.,

$$
\begin{aligned}
& \left(b-1-v_{j, n-1}\right) \ldots\left(b-1-v_{j, 1}\right)\left(b-1-v_{j, 0}\right)= \\
& =A\left(v_{j, n-1} \ldots v_{j, 1} v_{j, 0}\right)= \\
& =\left(b-1-v_{j, 0}\right)\left(b-1-v_{j, 1}\right) \ldots\left(b-1-v_{j, n-1}\right) .
\end{aligned}
$$

Consequently, $m$ is antipalindromic in base $b$ if and only if $\left(u_{j}\right)_{b}=\overline{v_{j, n-1} \cdots v_{j, 1} v_{j, 0}}$ is a palindrome for all $j \in\{0,1, \ldots, k\}$.

Example 6. Consider $m=73652$. Then $(m)_{27}=$ $\overline{(3)(20)(6)(23)}=\overline{u_{3} u_{2} u_{1} u_{0}}$, thus, $m$ is antipalindromic in base $27=3^{3}$. However, $(m)_{3}=$ $\overline{10202020212}$, thus $m$ is not antipalindromic in base 3. If we cut $(m)_{3}$ into blocks of length 3 , then all of them are palindromic. However, the first one equals 010 and it starts with a zero, hence the assumption $u_{3} \geq 9$ of Theorem 15 is not met.

Example 7. Consider $b=10$. The number 6633442277556633 is an antipalindromic number both in base 10 and 100 .

\section{Conclusion And open PROBlems}

In this paper, we carried out a thorough study of antipalindromic numbers and described known results regarding palindromic numbers in order to draw a comparison. It brings a number of new results:

- We described the divisibility of antipalindromic numbers and showed that non-trivial antipalindromic primes may be found only in base 3 .

- We found several classes of antipalindromic squares and higher powers.

- We described pairs of bases such that there is a number antipalindromic in both of these bases. Moreover, we obtained the following interesting results concerning multi-base antipalindromic numbers: $\triangleright$ For any composite number, there exist at least two bases such that this number is antipalindromic in both of them.

$\triangleright$ For every $n \in \mathbb{N}$, there exist infinitely many numbers that are antipalindromic in at least $n$ bases.

$\triangleright$ Let $b \in \mathbb{N}, b \geq 2$. Then there exists $m \in \mathbb{N}$ such that $m$ is antipalindromic in base $b$ and in at least one more base less than $m$.

This paper is based on the bachelor thesis [14, where several more results were obtained:

- the number of (anti)palindromic numbers of a certain length and the maximum and minimum number of antipalindromic numbers between palindromic numbers and vice versa;

- an explicit formula for the length of gaps between neighboring antipalindromic numbers.

We created a user-friendly application for all the questions studied [9, which is freely available to the reader. Based on computer experiments, we state the following conjectures and open problems:

(1.) Are there infinitely many antipalindromic primes in base 3? (We know there is never more than one antipalindromic prime in any other base except for 3.) During an extended search, the first 637807 antipalindromic primes have been found.

(2.) We conjecture it is possible to express any integer number (except for 24, 37, 49, 117, and 421) as the sum of at most three antipalindromic numbers in base 3 . Our computer program shows that the answer is positive up to $5 \cdot 10^{6}$.

(3.) We conjecture it is possible to express any palindromic number in base 3 as the sum of at most three antipalindromic numbers in base 3 . This conjecture follows evidently from the previous one, and we verified it even for larger numbers, up to $10^{8}$.

(4.) Is there a pair of bases such that it is impossible to find any number that has an antipalindromic expansion in both of them? According to our computer experiments, suitable candidates seem to be the bases 6 and 8 . It is to be studied in the future.

\section{ACKNOWLEDGEMENTS}

We would like to thank to the reviewers for their useful comments and suitable suggestions concerning references. L. Dvořáková received funding from the Ministry of Education, Youth and Sports of the Czech Republic through the project no. CZ.02.1.01/0.0/0.0/16_019/0000778.

\section{REFERENCES}

[1] J. Joyce. Ulysses. First edition. Shakespeare and Company, 12, Rue de l'Odéon, Paris, 1922.

[2] N. J. A. Sloane. The on-line encyclopedia of integer sequences. https://oeis.org.

[3] A. Tripathi. Characterization and enumeration of palindromic numbers whose squares are also palindromic. Rocky Mountain J Math 50(3):1115-1124, 2020. https://doi.org/10.1216/rmj.2020.50.1115. 
[4] G. J. Simmons. On palindromic squares of non-palindromic numbers. J Recreational Math 5(1):11-19, 1972.

[5] G. J. Simmons. Palindromic powers. J Recreational Math 3:93-98, 1970.

[6] W. D. Banks, D. N. Hart, M. Sakata. Almost all palindromes are composite. Math Res Lett 11(5-6):853-868, 2004. https://doi.org/10.4310/MRL.2004.v11.n6.a10

[7] J. Cilleruelo, F. Luca, L. Baxter. Every positive integer is a sum of three palindromes. Math Comp 87(314):30233055, 2018. https://doi.org/10.1090/mcom/3221

[8] A. Rajasekaran, J. Shallit, T. Smith. Additive number theory via automata theory. Theory Comput Syst 64(3):542-567, 2020.

https://doi.org/10.1007/s00224-019-09929-9
[9] S. Kruml. Antipalindromic numbers (application). [2020-08-10], https :

//github.com/Kruml3/Antipalindromic-numbers/.

[10] J. Cilleruelo, F. Luca, I. E. Shparlinski. Power values of palindromes. J Comb Number Theory 1(2):101-107, 2009.

[11] B. Bašić. On $d$-digit palindromes in different bases: the number of bases is unbounded. Int $J$ Number Theory 8(6):1387-1390, 2012. https://doi.org/10.1142/S1793042112500819

[12] B. Bašić. On "very palindromic" sequences. J Korean Math Soc 52(4):765-780, 2015. https://doi.org/10.4134/JKMS.2015.52.4.765.

[13] A. Bérczes, V. Ziegler. On simultaneous palindromes. J Comb Number Theory 6(1):37-49, 2014.

[14] S. Kruml. Antipalindromic numbers. bachelor thesis, Czech Technical University in Prague, 2020. Available on request. 